\title{
Adopting Different Quality Parameter Settings to increase GSM Voice Capacity by using Voice Service over Adaptive Multi-user Channels on One Slot (VAMOS) in Asiacell in Baghdad Network
}

\author{
Mohammed Kais Afar \\ University of Baghdad
}

Electronics \& Communications Eng. Dept. BaghdadIraq

\begin{abstract}
This Paper aims to study VAMOS Hardware Efficiency for different quality setting and fixing all other parameters in live network in Asia cell Telecommunication Company in Baghdad, check the impact of VAMOS activation in all major KPI (Key Prediction Indicators) and choose the better setting with minimum KPI degradation.
\end{abstract}

\section{General Terms}

Increasing voice channel capacity for GSM networks.

\section{Keywords}

GSM, OSC, VAMOS, QPSK

\section{INTRODUCTION}

The rapid growth of the GSM subscriber base has led to aggressive network expansion, especially in emerging market. Telecom operators need to increase spectrum utilization of voice service and improve hardware efficiency of both new \& already installed network equipment. The advent of new wireless service makes these needs even more acute. [1]

VAMOS is introduced in the GSM EDGE Radio Access Network (GERAN) evolution. It is developed on the basis of Multi-User Reusing One Slot (MUROS). The VAMOS feature expands the GERAN network capacity without adding transceivers (TRXs) or frequencies.

The key VAMOS technique for the GSM network is the multiplexing of two users onto the same channel, as illustrated in Figure 1. At present, VAMOS, implemented in Huawei GBSS products, enables two users to be multiplexed on the same half-rate (HR) channel .[2, 3,4,5,6]

To enable VAMOS, MSs (mobile station) must support VAMOS. At present, the MSs that support VAMOS are classified as None-SAIC (Single Antenna Interference Cancelation) MS, SAIC MS, VAMOS-1 MS, and VAMOS-2 MS. VAMOS significantly increases the capacity of a network with loose frequency reuse and a high percentage of VAMOS-capable MSs . [2,3,4,5,6]

\author{
Yamaan E. Majeed, PhD. \\ University of Baghdad
}

Electronics \& Communications Eng. Dept. BaghdadIraq

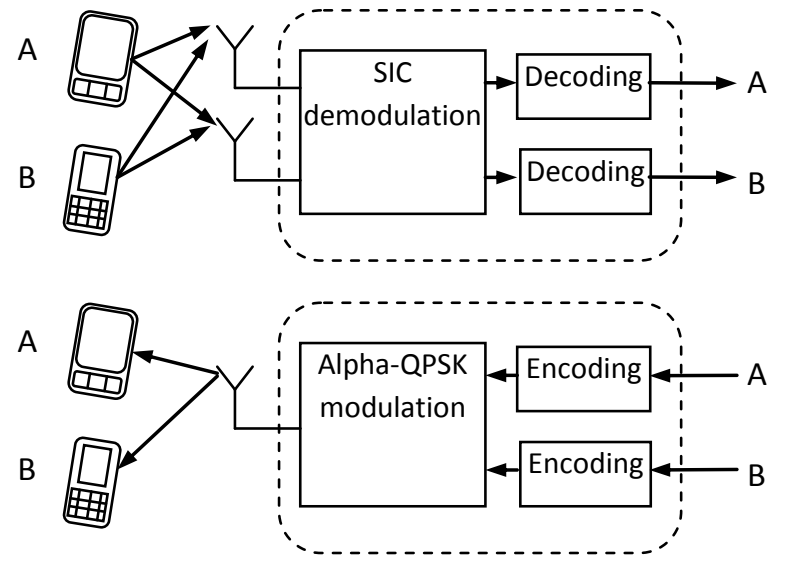

Fig. 1: Multiplexing Techniques of VAMOS

\section{MODULATION AND DEMODULATION}

\subsection{Uplink SIC (Successive Interference Cancelation) Demodulation}

VAMOS uses SIC as the uplink demodulation algorithm. The BTS (Base Station Transceiver) receives information from two users on the same HR channel, obtains the data to be decoded and the information in the measurement reports using high- and low-power user demodulation, and then decodes their respective data.

SIC first uses the Interference Rejection Combining (IRC) algorithm to demodulate the signals of the high-power user to obtain its data through channel decoding, and then subtracts the high-power user signals from the total received signals, and finally performs IRC demodulation on the signals of the low-power user to obtain its data through channel decoding.[2]

Uplink SIC demodulation requires that there be a power difference between the two multiplexed users. Therefore, their power needs to be adjusted accordingly using VAMOS uplink power control. 


\subsection{Downlink QPSK Modulation}

VAMOS uses QPSK (Quadrature Phase Shift Keying) as the downlink modulation algorithm. QPSK supports VAMOS multiplexing through handover and can adjust the modulation method of an ongoing call.

In QPSK modulation, the power of the two multiplexed users can be adjusted. Downlink QPSK demodulation requires that there be a power difference between two multiplexed users. Therefore, their power needs to be adjusted accordingly using VAMOS downlink power control. [2]

\section{MULTIPLEXING AND DEMULTIPLEXING}

VAMOS multiplexing multiplexes two suitable users onto one HR (Half Rate) channel by using channel assignment or intracell handover. VAMOS multiplexing expands network capacity and solves the problem of resource insufficiency without adding TRXs or frequencies.

After VAMOS multiplexing is performed, multiplexed users in the cell are demultiplexed and handed over to idle HR channels if the speech quality deteriorates or the cell load is lower than or equal to a specified threshold.

In the VAMOS multiplexing procedure, two decisions are made: which candidate VAMOS user to select and whether to trigger VAMOS multiplexing. Figure 2 shows the VAMOS multiplexing procedure. [2]

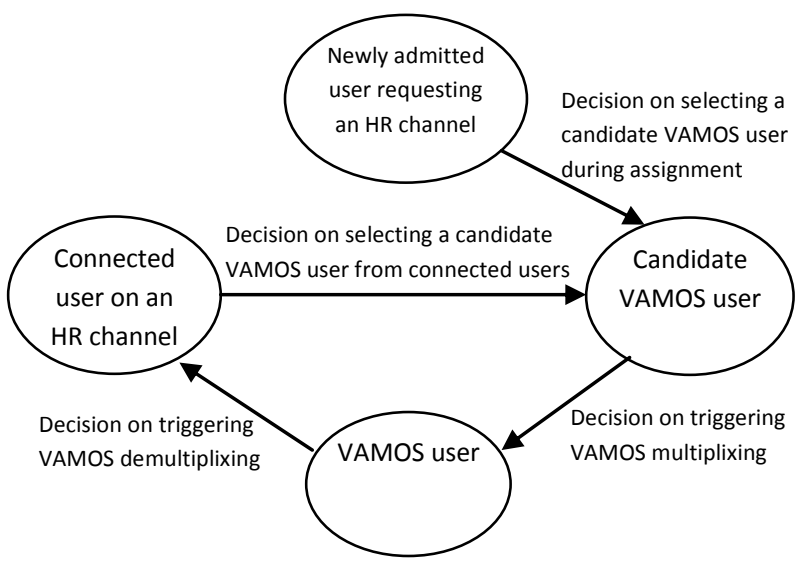

Fig. 2: Multiplexing - Demultiplexing

\section{HARDWARE EFFICIENCY (HDE)}

Hardware efficiency is the percentage of VAMOS traffic out of total Traffic carried by the cell. [2,7]

\section{Hardware efficiency $=(100 *$ VAMOS Traffic/Total Traffic $)$}

It is an indicator shows how many TRXs (Transceiver unit) can be dispensed. In case there is a cell with 4 TRXs, and there is HW efficiency $=25 \%$, that means traffic of 3TRXs with VAMOS $=$ traffic of 4 TRXs without VAMOS, which means:

1. One carrier can be dispensed which will improve spectrum utilization which is very important point in GSM as frequency band is limited.

2. One TRX can be dispensed which reduce cost.
3. Faster solution to overcome $\mathrm{TCH}$ congestion as compared to add TRX.

\section{VAMOS GAIN}

VAMOS Gain represents VAMOS user's percentage. It can be displayed by the number of VAMOS users or the percentage of VAMOS traffic volume to the overall traffic volume. Theoretically, it should be equal to Hardware efficiency $[2,7]$.

\section{VAMOS Gain $(\%)$ = VAMOS Area $(\%)$ x SAIC Ratio $(\%)$ $x$ HR Ratio (\%)}

\subsection{Calculating SAIC Ratio (\%):}

It is the Number of Calls Originated or Terminated by MSs Supporting SAIC out of total number of calls originated for the same time which can be collected from the system. Collect one week's data for traffic statistics on the system. Then calculate the SAIC Ratio by using the following formula[2,7,8,9]:

SAIC Ratio $(\%)=($ Number of Calls Originated or Terminated by MSs Supporting SAIC)/( Number of Calls) $\times 100 \%$.

Figure 3 shows SAIC capability measured in Asiacell network. The Figure shows that there is good SAIC capability in the network which produces good chance to get high number of VAMOS users in the network.

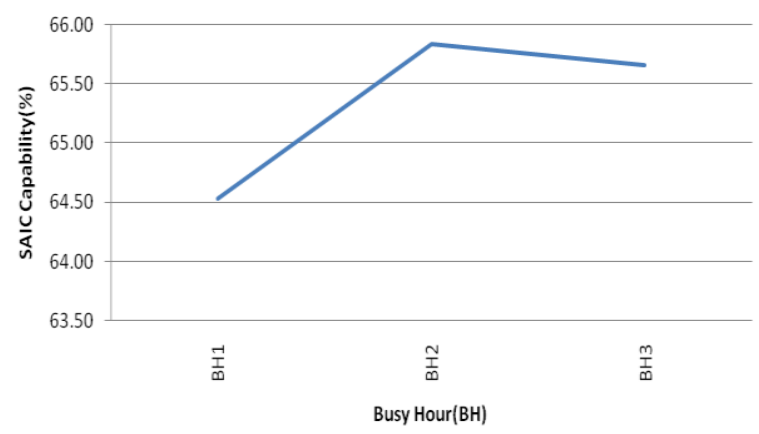

Fig 3: SAIC Capability in the network

\subsection{Calculating HR Ratio (\%):}

The proportion of both TCHHs (Traffic Channel Half Rate) and AMR (adaptive multi-rate) HR channels out of total Traffic which is calculated using as below after extracting all related measurement from the system $[2,6,7,8,9]$ :

\section{HR Ratio $(\%)=($ TCHH Traffic Volume $) /($ Traffic Volume on TCH)}

Figure 4 shows HR ratio in the network. The figure shows there is high HR ratio in these sites which means there is high possibility to deploy VAMOS because VAMOS work only in 
HR channels. HR ratio was low on week end on $6^{\text {th }}$ and $7^{\text {th }}-$ Jan-2013.

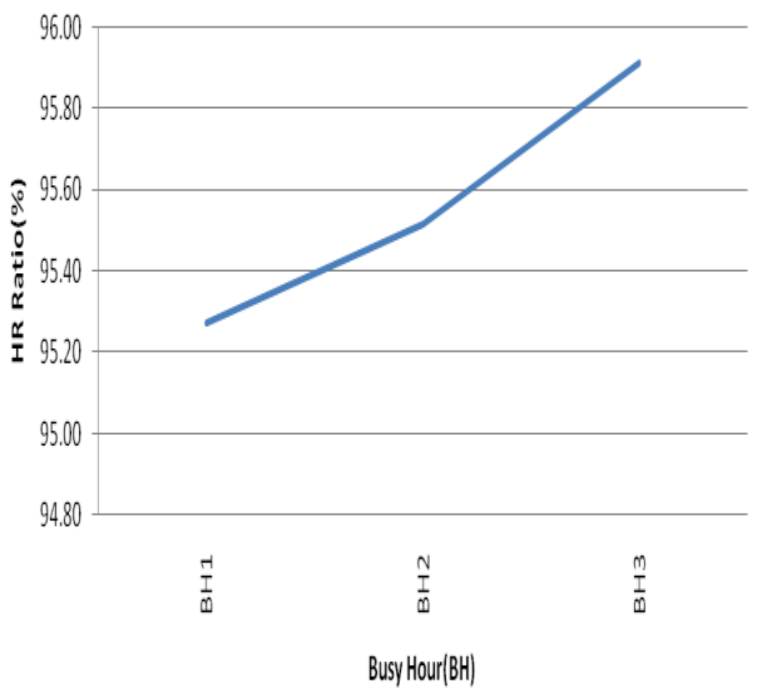

Fig 4: HR Ratio

\subsection{Calculating VAMOS Area (\%):}

VAMOS Area (\%) represents the area under the BSC that meets both VAMOS Multiplexing Quality Threshold and VAMOS Multiplexing Level Threshold. The received level ranges from rank 0 to rank 7 . Each rank corresponds to a received level range. Higher rank suggests higher receive level. The following shows the relationship between ranks and receive level ranges:

Rank 0: receive level $\leq-100 \mathrm{~dB}$, Rank 1: receive level (-100, -95) dB , Rank 2: receive level (-95, -90) dB ,Rank 3: receive level (-90, -85) dB , Rank 4: receive level $(-85,-80) \mathrm{dB}$, Rank 5: receive level $(-80,-75) \mathrm{dB}$, Rank 6: receive level $(-75,-70)$ dB , Rank 7: receive level > $-70 \mathrm{~dB}$.

The receive quality ranges from rank 0 to rank 7 . Each rank corresponds to a bit error rate (BER) range. Higher rank suggests higher BER and worse receive quality. The following shows the relationship between receive quality ranks and BER ranges:

Rank 0: BER < $0.2 \%$, Rank 1: BER [0.2\%, 0.4\%), Rank 2: BER $[0.4 \%, 0.8 \%)$, Rank 3: BER [0.8\%, 1.6\%), Rank 4: BER $[1.6 \%, 3.2 \%)$, Rank 5: BER [3.2\%, 6.4\%), Rank 6: BER $[6.4 \%, 12.8 \%$ ), Rank $7:$ BER $\geq 12.8 \%$

All previous measurements had been extracted from the system and then VAMOS Area can be calculated as follow $[2,3,7,8,9]$ :

VAMOS Area $(\%)=[$ Number of MRs on TCHH and TCHF (Receive Quality Rank 0 or 1 and Receive Level Rank 4, 5, 6 or 7)]/[Number of MRs on TCHH and TCHF] $\mathrm{x} 100 \%$

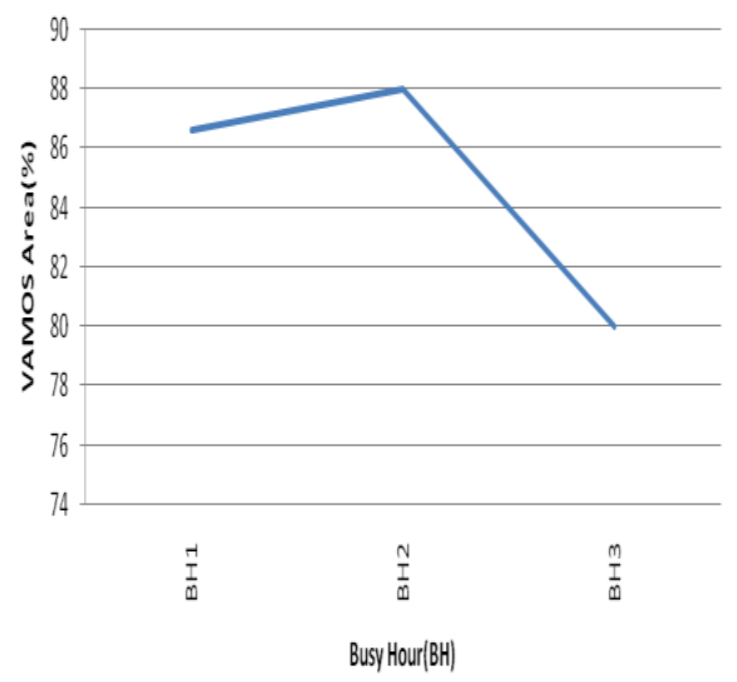

Fig 5: VAMOS Area

Figure 5 shows that there is high percent of quality measurements in high range (less than or equal to 1), i.e. BER (Bit Error Rate) less than or equal to $0.4 \%$, which produce high possibility to apply VAMOS.

\section{NETWORK DESIGN AND IMPLEMENTATION}

14 sites were chosen in Asia cell Company for mobile communication in Baghdad. Three tests were implemented in these sites; all parameters were kept to default $[9,10,11,12$, $13,14,15]$, except quality threshold. A cluster of sites that has been chosen have cells with high traffic which is needed to trigger VAMOS.

All the sites names in the selected cluster are shown in table1.

Table 1: Site Name

\begin{tabular}{|c|c|c|}
\hline \multicolumn{3}{|c|}{ Site Name } \\
\hline Khalani_2014 & Wathbasq_2416 & QanberAli_2154 \\
\hline Rusafi_2116 & Midan_2890 & Sadria_2389 \\
\hline Khulafa_2117 & QasimSeq_2902 & Alfadhel_2321 \\
\hline ElRashid_2121 & Sinak_2936 & MathamUniv_2672 \\
\hline Shorja_2960 & Zubaida_6199 & \\
\hline
\end{tabular}

Increasing quality threshold means increasing the numbers of VAMOS candidates, i.e. increasing the VAMOS traffic and Hardware Efficiency.

Table 2 shows the setting of different in the network with a description for each parameter. These parameters have been set to different values in each test $[9,10,11,12,13,14,15]$.

\section{RESULTS}

After implementing all quality tests as indicated in table 2, Hardware Effecieny for two BH(Busy Hour), VAMOS Gain and DL Quality(5 7)\% are calculated after extracting VAMOS traffic and all related measurements from the system. 
Table 2: Parameters Setting for Three Tests

\begin{tabular}{|c|c|c|c|c|}
\hline Parameter & Description & Test1 & Test2 & Test3 \\
\hline $\begin{array}{c}\text { DL Rx Quality } \\
\text { Threshold of } \\
\text { Established Calls }\end{array}$ & $\begin{array}{l}\text { Threshold of the downlink receive quality of an established } \\
\text { call to be selected as a VAMOS candidate call. The } \\
\text { decision on this call can be triggered successfully only } \\
\text { when the following conditions are met: The ATCB of this } \\
\text { call is greater than or equal to the ATCB threshold. The } \\
\text { downlink receive quality is lower than or equal to the value } \\
\text { of this parameter. The uplink receive quality is lower than } \\
\text { or equal to "UL Rx Qual. Threshold. Of Established Calls". } \\
\text { If the decision conditions are met for "Duration of } \\
\text { Satisfying Candidate VAMOS Call" within "Watch Time } \\
\text { of Candidate Calls", this call can be selected as a VAMOS } \\
\text { candidate call. }\end{array}$ & 10 & 30 & 50 \\
\hline $\begin{array}{c}\text { UL Rx Quality } \\
\text { Threshold of } \\
\text { Established Calls }\end{array}$ & $\begin{array}{l}\text { Threshold of the uplink receive quality of an established } \\
\text { call to be selected as a VAMOS candidate call. The } \\
\text { decision on this call can be triggered successfully only } \\
\text { when the following conditions are met: The ATCB of this } \\
\text { call is greater than or equal to the ATCB threshold. The } \\
\text { uplink receive quality is lower than or equal to the value of } \\
\text { this parameter. The downlink receive quality is lower than } \\
\text { or equal to "DL Rx Qual. Threshold of Established Calls". } \\
\text { If the decision conditions are met for "Duration of } \\
\text { Satisfying Candidate VAMOS Call" within "Watch Time } \\
\text { of Candidate Calls", this call can be selected as a VAMOS } \\
\text { candidate call. }\end{array}$ & 10 & 30 & 50 \\
\hline $\begin{array}{c}\text { UL RX Bad Quality } \\
\text { Demultiplex } \\
\text { Threshold }\end{array}$ & $\begin{array}{l}\text { Uplink receive quality threshold of a VAMOS call in } \\
\text { channel demultiplexing due to poor speech quality in a cell. } \\
\text { When the uplink receive quality is higher than or equal to } \\
\text { this threshold or the downlink receive quality is higher than } \\
\text { or equal to "DL RX Poor Qual. Demultiplex Threshold.", } \\
\text { the decision of channel demultiplexing due to poor speech } \\
\text { quality is triggered. If the decision conditions are met for } \\
\text { "Poor Qual. Duration for Demultiplex" within "Watch } \\
\text { Time of Poor Qual. for Demultiplex", a call can be } \\
\text { demultiplexed through handover. }\end{array}$ & 30 & 60 & 70 \\
\hline $\begin{array}{c}\text { DL RX Bad Quality } \\
\text { Demultiplex } \\
\text { Threshold }\end{array}$ & $\begin{array}{l}\text { Downlink receive quality threshold of a VAMOS call in } \\
\text { channel demultiplexing due to poor speech quality in a cell. } \\
\text { When the downlink receive quality is higher than or equal } \\
\text { to this threshold or the uplink receive quality is higher than } \\
\text { or equal to "UL RX Poor Qual. Demultiplex Threshold ", } \\
\text { the decision of channel demultiplexing due to poor speech } \\
\text { quality is triggered. If the decision conditions are met for } \\
\text { "Poor Qual. Duration for Demultiplex" within "Watch } \\
\text { Time of Poor Qual. for Demultiplex", a call can be } \\
\text { demultiplexed through handover. }\end{array}$ & 30 & 60 & 70 \\
\hline
\end{tabular}


Figure 6 shows the Hardware Effecicncy versus Downlink Quality(5 7) from these tests.

Form Figure 6, below points can be concluded:

1.VAMOS Gain is more than $50 \%$, which a very good opportunity to get high Hardware Efficiency, i.e. more TRXs can be dispensed to improve spectrum utilization.

2.Hardware efficiency is ranged from $42 \%$ to $33 \%$ for different quality settings which is fantastic results as it is near to VAMOS Gain, also it means that most of calls lies within very good quality (less than or equal to 1) which means there is minimum quality degradation, so Test $(1,3)$ is the better setting.

3. There is an increment in Downlink Quality (5 7) in Test $(3,5)$ due to TRX fluctuation in three sites which is still acceptable.

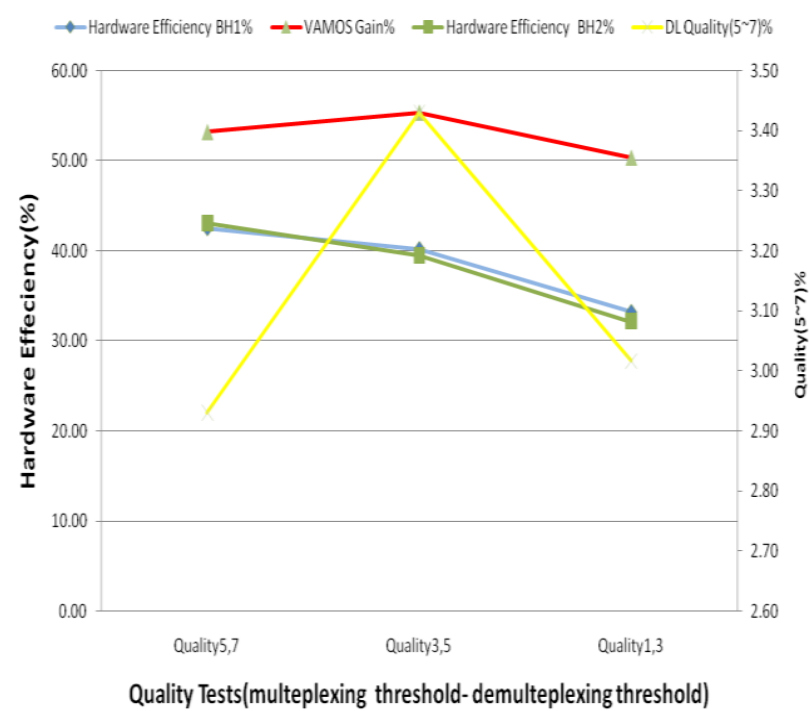

Fig 6: Hardware Effciency-DL Quality(5 7) for different Quality setting.

Figure 7 shows CSSR(Call Setup Sucees Rate) in two BH(Busy Hour) measurement, small degridation in CSSR had been happened during Test $(3,5)$ and Test $(1,3)$ because three sites got out of services for short time and came back to service that lead to these slitgh derement, i.e. these degredation was not related to VAMOS implemantation.

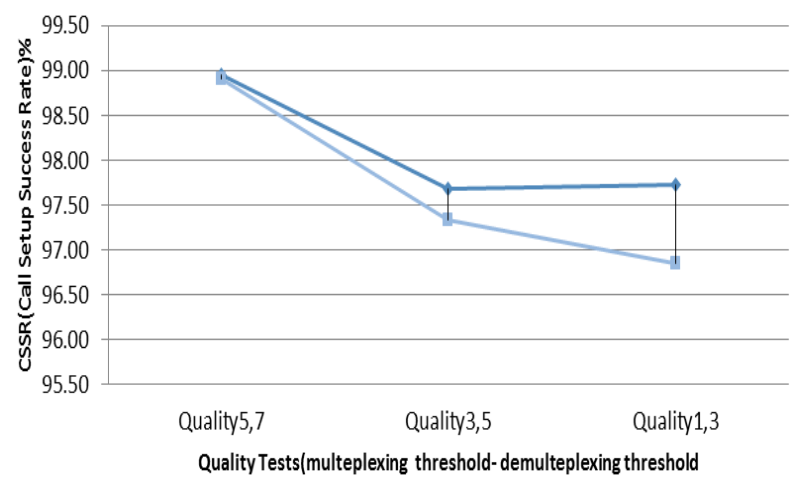

Fig 7: CSSR for different quality setting
As number of MSs(mobile station handset) with bad quality is low, so low number of multiplexing process (i.e. VAMOS traffic) will happened, so possibility of quality degradation will be low, so that CDR\% still good as shown in Figure 8.

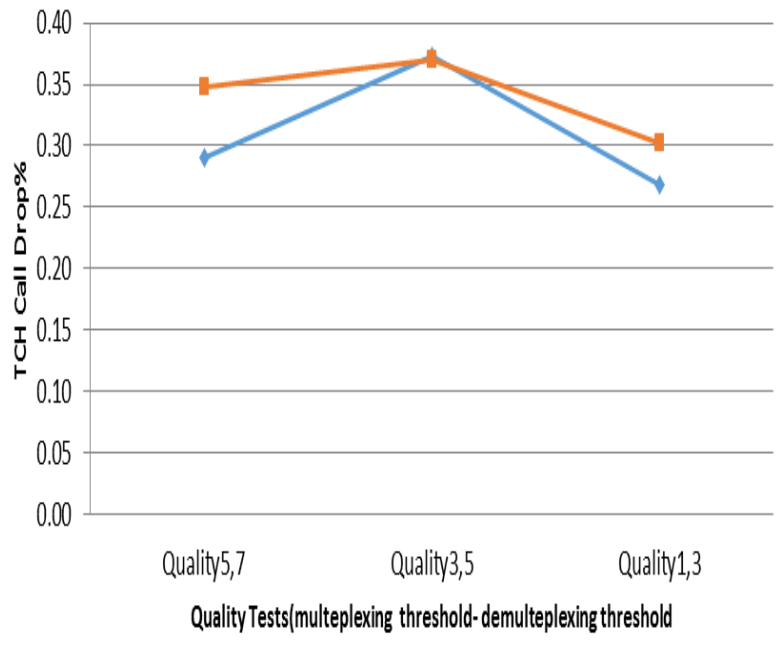

Fig 8: CDR(Call Drop Rate)\% for different quality setting

\section{CONCLUSIONS}

VAMOS is promising technology to enhance capacity of voice service of the GSM/EDGE networks in ASIA Cell Company in Baghdad as below:

1. High VAMOS Gain is existed in the network which produces promising possibility to apply VAMOS technique.

2. High Hardware Efficiency is obtained by applying different quality setting.

3. High number of TRX can dispensed which will improve spectrum utilization and reduce frequency clashes which will improve quality.

4. As many TRXs will be dispensed, Reframing some of the spectrum they are using for $2 \mathrm{G}$ services for $3 \mathrm{G}$ services can be implemented.

5. Better quality setting with good hardware efficiency is Test3, which is highly recommended to be default setting in the network.

\section{REFERENCES}

[1] Ericsson white paper "DOUBLE GSM VOICE CAPACITY”, www. Ericsson.com, July 2010

[2] Huawei Technologies Library"VAMOS GBSS14.0 Feature Parameter Description", Huawei Technologies Co., Ltd., 2011.

[3] Meik Kottkamp "VAMOS Technology Introduction, Application Note" Rohde \& Schwarz GmbH \& Co. KG , 2010.

[4] Jyrki T.J. Penttinen1, Francesco Calabrese1, Kari Niemelä2 , David Valerdi, Maria Pilar Molina "Performance Model for Orthogonal Sub Channel in Noise-limited Environment" ,Sixth International Conference on Wireless and Mobile Communications , 2010 
[5] D. Molteni , M. Nicoli , M. Säily "Resource Allocation Algorithm for GSM-OSC Cellular Systems", This full text paper was peer reviewed at the direction of IEEE Communications Society subject matter experts for publication in the IEEE ICC 2011 proceedings , 2011.

[6] Nokia Siemens Networks "Doubling GSM voice capacity with the Orthogonal Sub Channel" 2009 Nokia Siemens Networks, 2009.

[7] Huawei Technologies Library "VAMOS Deployment Guide (GBSS R13)" , Huawei Technologies Co., Ltd. 2011

[8] Huawei Technologies Library " Half-Rate Service Feature Parameter Description “ , Huawei Technologies Co., Ltd. 2011

[9] Huawei Technologies Library "Channel Management Feature Parameter Description " Huawei Technologies Co., Ltd. 2011
[10] Huawei Technologies Library "Handover Feature Parameter Description" Huawei Technologies Co., Ltd. 2011

[11] Huawei Technologies Library "Power Control Feature Parameter Description" Huawei Technologies Co., Ltd. 2011

[12] Huawei Technologies Library "BSC6900 Feature List "Huawei Technologies Co., Ltd. 2011

[13] Huawei Technologies Library "BSC6900 Optional Feature Description" Huawei Technologies Co., Ltd. 2011

[14] Huawei Technologies Library "BSC6900 GSM Parameter Reference" Huawei Technologies Co., Ltd. 2011

[15] Huawei Technologies Library "BSC6900 GSM MML Command Technologies Co., Ltd. 2011.
Huawei 